\title{
Study of the Self-Stabilization Ability of Tzatziki (a Traditional Greek Ready-to-Eat Deli Salad)
}

\author{
Stavros Lalas ${ }^{\mathrm{a}}$, Vassilis Athanasiadis ${ }^{*}$, Ioanna Karageorgou $^{\mathrm{a}}$, Eleni Bozinou ${ }^{\mathrm{b}}$, \\ And VAssilis G. Dourtoglou ${ }^{\mathrm{c}}$ \\ ${ }^{\text {a }}$ Department of Food Technology, Technological Educational Institution of Thessaly, Terma N. Temponera \\ str., GR-43100, Karditsa, Greece \\ b Department of Agricultural Technology, Technological Educational Institution of Western Macedonia, Terma \\ Kontopoulou str., GR-53100, Florina, Greece \\ ${ }^{\mathrm{c}}$ Department of Wine, Vine and Beverage Sciences, University of Western Attica, Agiou Spyridonos str., \\ GR-12210, Egaleo, Athens, Greece \\ ${ }^{*}$ Corresponding author \\ vs.athanas@teilar.gr \\ TEL: $+30-24410-64783$ \\ FAX: $+30-24410-64781$
}

Received: 13 May 2018; Published online: 18 April 2019

\begin{abstract}
Traditional Greek yogurt-based salad Tzatziki is one of the most popular ready-to-eat deli salads in Greece. The objective of this study was to estimate the microbial stability of Tzatziki, with and without chemical preservatives, using a rapid method. Determination of the microbial count was carried out using the bioluminescence method (ATP) and traditional microbiological analysis, plate-counting method (CFU) in various batches of the final product of Tzatziki. The results showed that the Tzatziki salad without preservatives initially gave higher relative light units (RLU) values $(79,532)$ than the same salad with preservatives $(43,198)$ because the potassium sorbate and the sodium benzoate, used the in recipe, appeared to suspend the action of microorganisms. After incubation in two different substrates, MacConkey and Sabouraud, the Tzatziki salad without preservatives gave higher RLU values $(9,488$ and 16,176 , respectively) than the salad with preservatives (12,780 and 12,005 , respectively). In the two selective substrates, differences appeared between the two methods of microbial count (RLU and CFU). While RLU values were roughly at the same level, the CFU values presented significant differences $(\mathrm{p}<$ $0.05)$. It was also shown that there was a strong correlation $\left(R^{2}=0.93-0.95\right)$ between bacterial counts estimated by traditional CFU and ATP methods. As expected, the dominant microbial population in Tzatziki was Lactobacillus spp., originated from yogurt. Coliforms and yeasts were not able to survive in this environment. Generally, according to the results, Greek traditional Tzatziki salad was a microbial stable product and the bioluminescence method could be a rapid method to determine its microbial state.
\end{abstract}

Keywords: Bioluminescence; Microbial stability; Tzatziki; Greek deli salad

\section{Introduction}

Tzatziki, one of the most popular Greek ready to eat deli salads, is widely consumed in Greece, the East Mediterranean region and Balkan countries, as well as in other parts of the world, including the rest of Europe and USA. Tzatziki is made of strained yogurt (usually from sheep or goat milk) mixed with fresh chopped cucumber, garlic, salt, olive oil, vinegar and dill. 
Tzatziki is a low $\mathrm{pH}$ food, due to the presence of yogurt and the added vinegar, and it has a low salt percentage, $2-3 \%$. This dressing has shown to be resistant to microbial spoilage by pathogen microorganisms (Skandamis, Davies, McClure, Koutsoumanis, \& Tassou, 2002). Tzatziki is generally recognized for its health properties and for more than a hundred years, people accompany meals rich in fat with this salad.

As far as yogurt is concerned, there is a tremendous scientific work related to its microbial stability. The same goes for garlic and garlic extract, with an emphasis on the effect of garlic on microorganisms. Garlic is considered a germicide (Harris, Cottrell, Plummer, \& Lloyd, 2001). Although, there is little documentation about cucumber's activity on microorganisms, it has been reported that the volatile oils of cucumber (Cucumis sativus) show antibacterial activity, as well as a minimum inhibitory concentration (MIC), 0.09-0.50 mg $\mathrm{mL}^{-1}$, against both gram-positive and gram-negative bacteria, and anti-fungal activity against human pathogen fungi (Candida albicans, C. tropicalis and $C$. glabrata $)\left(\mathrm{MIC}=1.34-2.90 \mathrm{mg} \mathrm{mL}^{-1}\right)$ (Sotiroudis, Melliou, Sotiroudis, \& Chinou, 2010). (E,Z)-2,6-nonadienal and $(E)$-2-nonenal have been found in cucumber and have shown strong activities to several human and foodborne pathogen bacteria such as Bacillus cereus, Escherichia coli O157:H7, Listeria monocytogenes and Salmonella typhimurium (Cho, Buescher, Johnson, \& Janes, 2004; Croft, Juttner, \& Slusarenko, 1993).

Conventional approaches to the determination of microbial stability rely on fermentation tests and cell counting methods. Among the relatively rapid methods for detection of microorganisms in food, adenosine triphosphate (ATP) bioluminescence is very suitable for on-line monitoring of bacterial contamination in food and beverages. This method does not require a culturing step or large equipment to fulfil the measurement, and it is fast and sensitive (Bottari, Santarelli, \& Neviani, 2015). Bioluminescence is proposed to be the technique giving results in the shortest time (Hawronskyj \& Holah, 1997). The ATP bioluminescence sensing assay relies on the fact that ATP is a major biological energy source existing in various microbes and, therefore, reflects the existence of living microbes (Luo et al., 2009).

The Celsis Lumac IMC kit, which was used in this research, was designed to detect the presence or absence of microorganisms in industrial samples and/or screen for microbial level contamination. Results are obtained within minutes when more than $10^{4}$ microorganisms per $\mathrm{mL}$ are present in the sample tested. Below this limit or when samples have high quenching levels, either a filtration step or an incubation step is required for the microbial enrichment of the sample.

The assay procedure is based on the ATP bioluminescence technique, which employs the light-producing system of the firefly to detect microorganisms. The test procedure consists of the selective extraction of non-microbial (somatic) ATP by a specific somatic cell extractant (NRS). The extractant makes the cell membrane permeable for molecules like ATP, which leak out. Microbial cells are not affected because they have cell walls. Extracted non-microbial ATP is hydrolysed by an ATPase (Somase). Microbial ATP is then measured in a Celsis Lumac Biocounter, after the addition of NRB and Lumit-PM. The amount of light produced correlates with the amount of ATP in the sample, shown as Relative Light Units (RLU).

The light measurement is based on the following reaction:

Luciferin $+\mathrm{ATP}+\mathrm{O}_{2} \frac{\text { Luciferase }}{\mathrm{Mg}^{2+}}$

Oxyluciferin $+\mathrm{AMP}^{*}+\mathrm{PPi}^{*}+\mathrm{CO}_{2}+$ Light

To our knowledge, Tzatziki has not been subjected to any study of its microbial flora except for some recent works on its stability against pathogen microorganisms (Tsiraki \& Savvaidis, 2014).

The aim of the current study is to develop a rapid and low cost ATP bioluminescence-sensing approach to determine the microbial stability of industrial Tzatziki samples. 


\section{Materials and Methods}

\section{$2.1 \quad$ Material}

The reagents (Lumit-PM, Lumit-Buffer, NRS, NRB, Somase) for the determination of the microbial population with the bioluminescence method were obtained from Lumac B.V. (Landgraaf, Netherlands). The reagents used for the determination of Total microbial count, Lactobacillus spp., yeast and coliforms (Total Plate Count agar, MRS agar, Malt Extract agar and Violet Red Bile agar) were obtained from Merck Ltd (Darmstad, Germany). The substrates used for the determination of Lactobacillus spp., yeasts and coliforms (Ringer solution, Sabouraud broth and MacConkey broth) were obtained from Merck Ltd (Darmstad, Germany).

\section{$2.2 \quad$ Tzatziki samples}

Tzatziki salad was made using a simple homemade recipe with ingredients purchased from the local market. Strained yogurt, cucumbers (peeled and diced), olive oil, cloves of garlic (peeled), vinegar, salt, and chopped fresh dill (at $60,20,9,4.5,3,2.5$ and $1 \%$ of the total weight of the product, respectively) were mixed in a food processor until well-combined. The mixture was divided in two equal batches. In the first batch, sodium benzoate $0.05 \%$ and potassium sorbate $0.05 \%$ were added as preservatives. No preservatives were added to the second batch. Each batch was then divided into $100 \mathrm{~g}$ sub-batches, which were then packed in sealed plastic containers. All the containers were stored at $5{ }^{\circ} \mathrm{C}$.

\subsection{Determination of the microbial population using firefly luciferase for ATP measurement}

The method used for the inoculation of salad is described in Fig. 1. The ATP assay was carried out as follows: $200 \mu \mathrm{L}$ of every sample was aseptically transferred to a cuvette; $100 \mu \mathrm{L}$ of Somase/NRS reagent was added to each sample and the samples were left for a $45 \mathrm{~min}$ period for incubation. Then, $100 \mu \mathrm{L}$ from each mixture were taken and placed into cuvettes. The cuvettes were inserted into the Celsis Lumac Biocounter 1800 (Landgraaf, Netherland) and the assay started. The instrument automatically added $100 \mu \mathrm{L}$ of NRB solution and the samples were left for $10 \mathrm{sec}$ in order for the extraction to take place. Finally, $100 \mu \mathrm{L}$ of Lumit-PM reagent was added to every sample.

\subsection{Determination of Colony Forming Units (CFU)}

The determination of CFU was carried out according to ISO 4833-1 (International Standard Organization, 2003a) standard methodology for the Total Plate Counts, ISO 21527-1 (International Standard Organization, 2008) for the enumeration of yeasts and moulds, ISO 5541-1 (International Standard Organization, 1986) for the enumeration of coliforms and ISO 9232 (International Standard Organization, 2003b) for the identification of yogurt Lactobacilli. Subsamples of $25 \mathrm{~g}$ of each Tzatziki sample were diluted in $225 \mathrm{~mL}$ of Ringer solution, homogenized with a stomacher (Bioscience International, $11607 \mathrm{Ma}$ gruder Lane, Rockville MD 20852-4365, USA), and then, incubated for $1 \mathrm{~h}$ at $25{ }^{\circ} \mathrm{C}$. Suitable 1:10 dilutions were prepared. The counting of Total Mesophilic Flora and Lactobacillus spp. was performed by pouring aliquots of the decimal dilutions in Total Plate Count agar and MRS agar, respectively. For yeast counting, 25 $\mathrm{g}$ of each sample was homogenized in $225 \mathrm{~mL}$ of Sabouraud broth and incubated at $35{ }^{\circ} \mathrm{C}$ for 24 h. Decimal dilutions of Sabouraud broth were prepared and poured in plates for total viable yeast counting, using Malt Extract agar. For coliform counting, $25 \mathrm{~g}$ of each sample was homogenized in $225 \mathrm{~mL}$ of MacConkey broth and incubated at $30^{\circ} \mathrm{C}$ for $24 \mathrm{~h}$. Decimal dilutions of MacConkey broth were also prepared and poured in plates for coliform counting, using Violet Red Bile agar. The incubation of Violet Red Bile agar samples was carried out at $30^{\circ} \mathrm{C}$ for $48 \mathrm{~h}$ and of Malt Extract agar at $25{ }^{\circ} \mathrm{C}$ for 5 days. After the incubation, the results were expressed in colony forming units (CFU) $\mathrm{g}^{-1}$ based on the average count of a triplicate determination. 


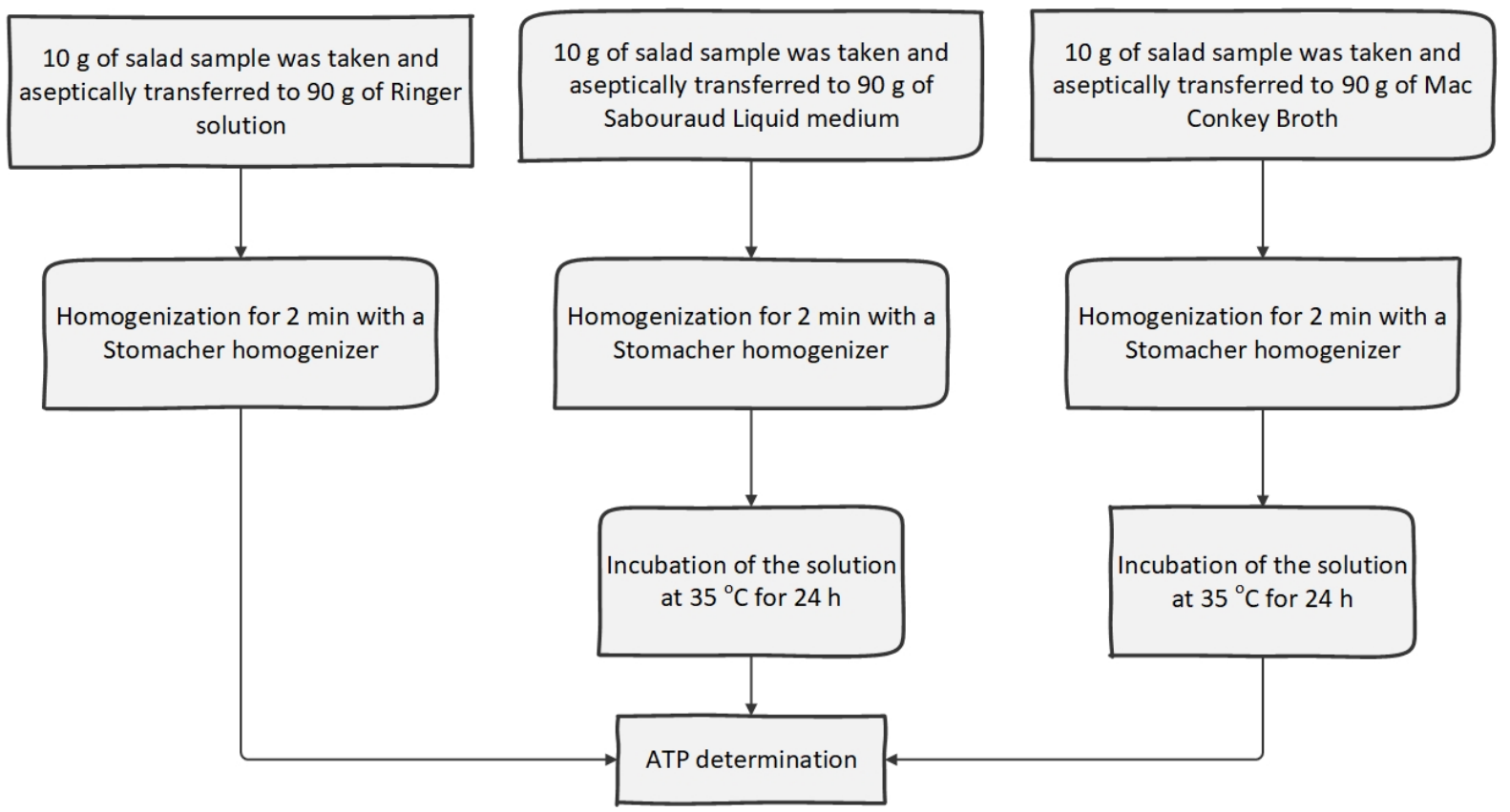

Figure 1: Method for the inoculation of salad

\subsection{Statistical analysis}

Results represent the average of three simultaneous assays. Statistical significance of the differences between mean values was assessed by ANOVA; $\mathrm{p}<0.05$ was considered as statistically significant.

\section{Results and Discussion}

The microbiological analyses were carried out on various batches of Tzatziki using, each time, three samples from each batch for each measurement in order to evaluate the reproducibility of the method. There was no significant difference between the different batches. The curves presented a similar behavior in all cases (batches). As expected, the Tzatziki salad without preservatives initially gave RLU values higher $(79,532)$ than the same salad with preservatives $(43,198)$. The potassium sorbate and the sodium benzoate, used in the recipe, appeared to suspend the action of microorganisms. In both cases, the RLU values decreased day after day, and 10 days later tended to zero (Fig. 2).
The same procedure took place after incubation for the two selective mediums, in order to have information about spoilage microorganisms. Incubation was carried out because the initial microbial count of yeasts and coliforms was lower than $10^{4}$ microorganisms per $\mathrm{mL}$ of sample. As expected, the Tzatziki salad without preservatives after incubation in two different substrates (Fig. 3), MacConkey and Sabouraud, gave RLU values higher $(9,488$ and 16,176$)$ than the salad with preservatives $(12,780$ and 12,005$)$, respectively. The microbial count in Sabouraud medium, which is selective for the growth of yeasts, compared to MacConkey (selective for coliforms) was higher because the cells of yeasts are bigger than those of bacteria and contain more ATP per cell and hence produce higher RLU values (Deligaris, 1981). The amount of ATP in microbial cells may differ, depending on their physiological state and types of cells present. For example, injured or starved microbial cells may contain approximately $10-30 \%$ of ATP present in healthy cells and yeast cells contain approximately 100 times more ATP than bacterial cells (Mendonça, Juneja, \& Daraba, 2014). Gener- 
$80 \mid$ Lalas et al.

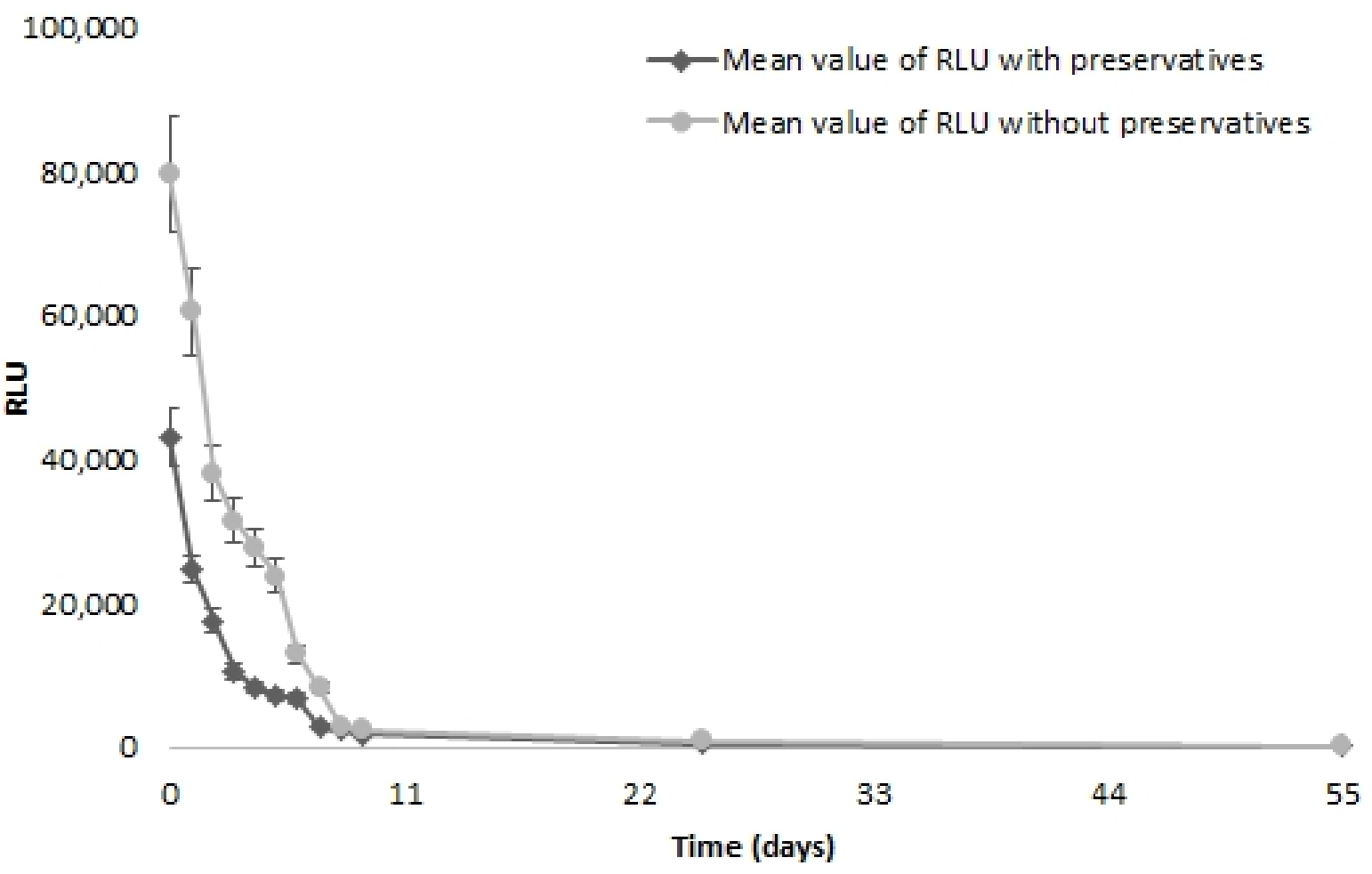

Figure 2: RLU values of Tzatziki, with and without preservatives (Ringer solution)

ally, coliforms, yeasts and fungi that existed in the system were given small possibility to grow due to the higher number of Lactobacilli present that function competitively against them. Thus, (Fig. 2) the decrease in population is progressive while the population of coliforms (Fig. 3a) and yeasts (Fig. 3b) decreases abruptly. The correlation between CFU and RLU measurements of Tzatziki, with and without preservatives, appear in Fig. 4.

In the two selective substrates, differences appeared between the two methods of microbial count (RLU and CFU) (Fig. 5). While RLU values were roughly at the same level, the CFU values presented significant differences $(\mathrm{p}<0.05)$. Particularly, the CFU values in the Sabouraud broth were initially lower than those in the MacConkey broth. This is explained by the size of the cells of yeasts that are bigger than those of the bacteria and contain more ATP per cell (Mendonça et al., 2014).
The correlation between RLU and CFU $\mathrm{g}^{-1}$ of salad in Ringer Solution, MacConkey and Sabouraud was almost linear in all cases.

In yogurt, generally because of the low $\mathrm{pH}$ value and the high number of Lactobacilli, the growth of other bacteria (proteolytical, coliforms) or yeasts is not being allowed. However, yogurt is sensitive to alterations from fungi, especially in the areas that are in contact with air. The microbial flora of Tzatziki is mainly composed of Lactobacilli, originated from yogurt (Tsiraki \& Savvaidis, 2014), and the results showed that Tzatziki salad presented an extreme stability from the $2^{\text {nd }}$ day of its preparation until the last day $\left(15^{\text {th }}\right)$ of experiments. Lactobacilli and other microorganisms were decreased and this was possibly due to the combination of ingredients (garlic, cucumber). Allicin and its derivative products (diallyl disulfide, diallyl trisulfide) which are found in garlic essential oils, have shown good antimicrobial activities 
Traditional Greek Tzatziki: Microbial stability | 81

(a) 20,000

$\longrightarrow$ Mean value of RLU (MacConkey) with preservatives

- Mean value of RLU (MacConkey) without preservatives

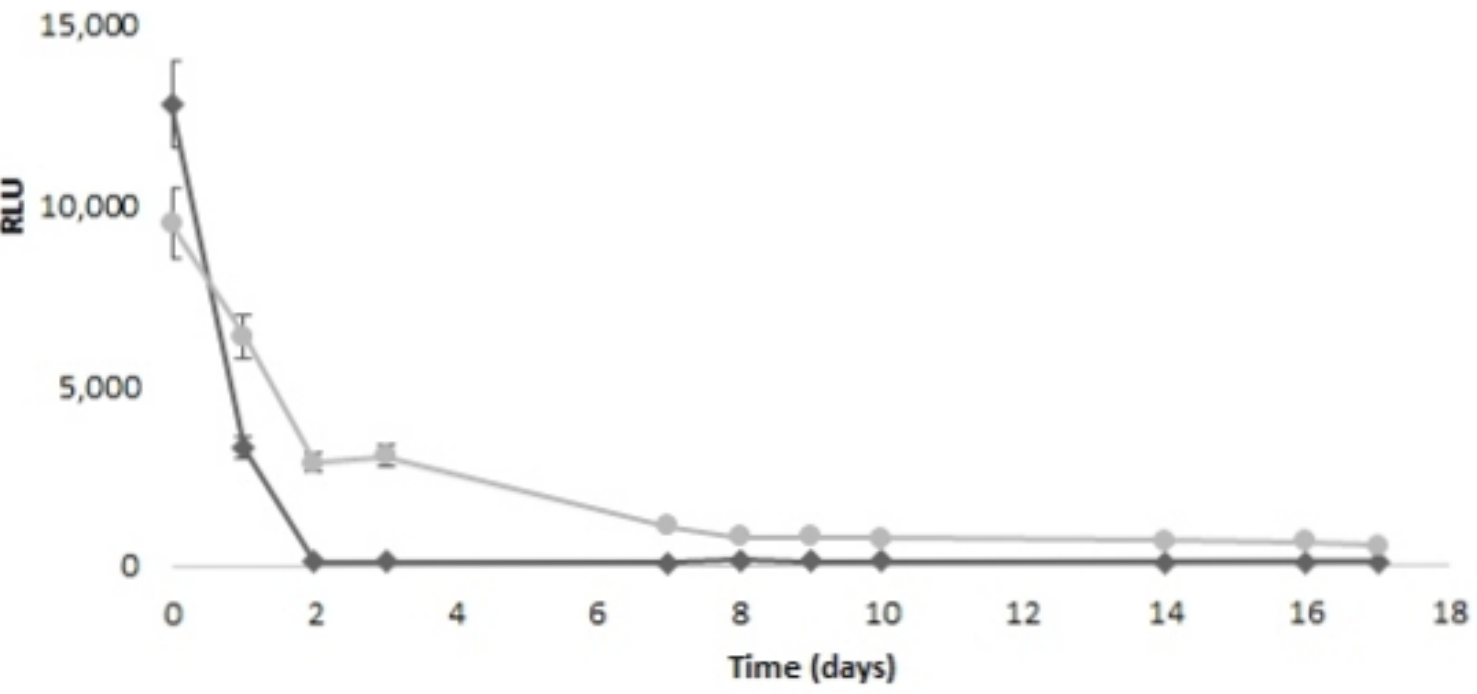

(b) 20,000

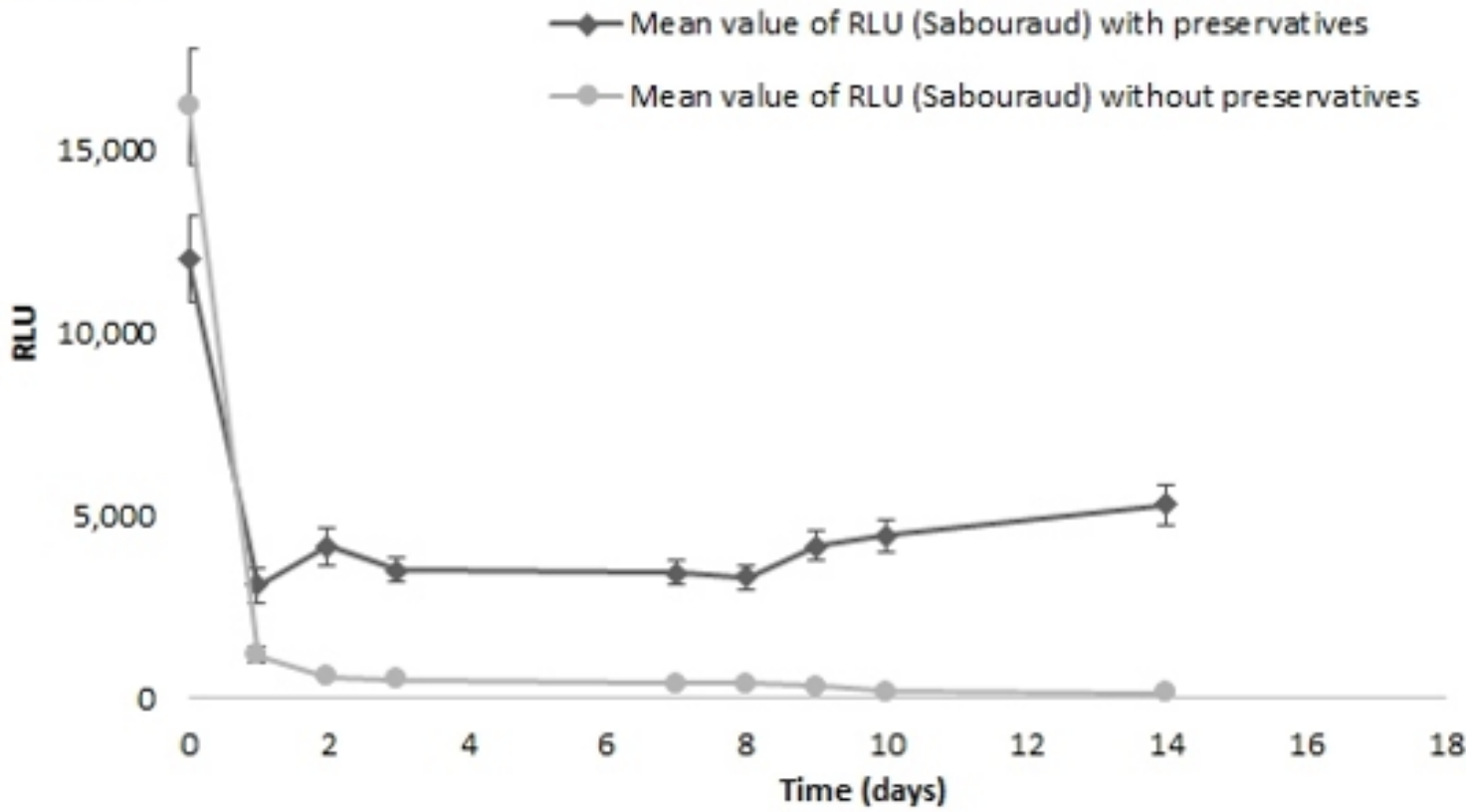

Figure 3: RLU values of Tzatziki, with and without preservatives, after incubation in (a) MacConkey and in (b) Sabouraud, for $24 \mathrm{~h}$ at $35^{\circ} \mathrm{C}$. 
$82 \mid$ Lalas et al.

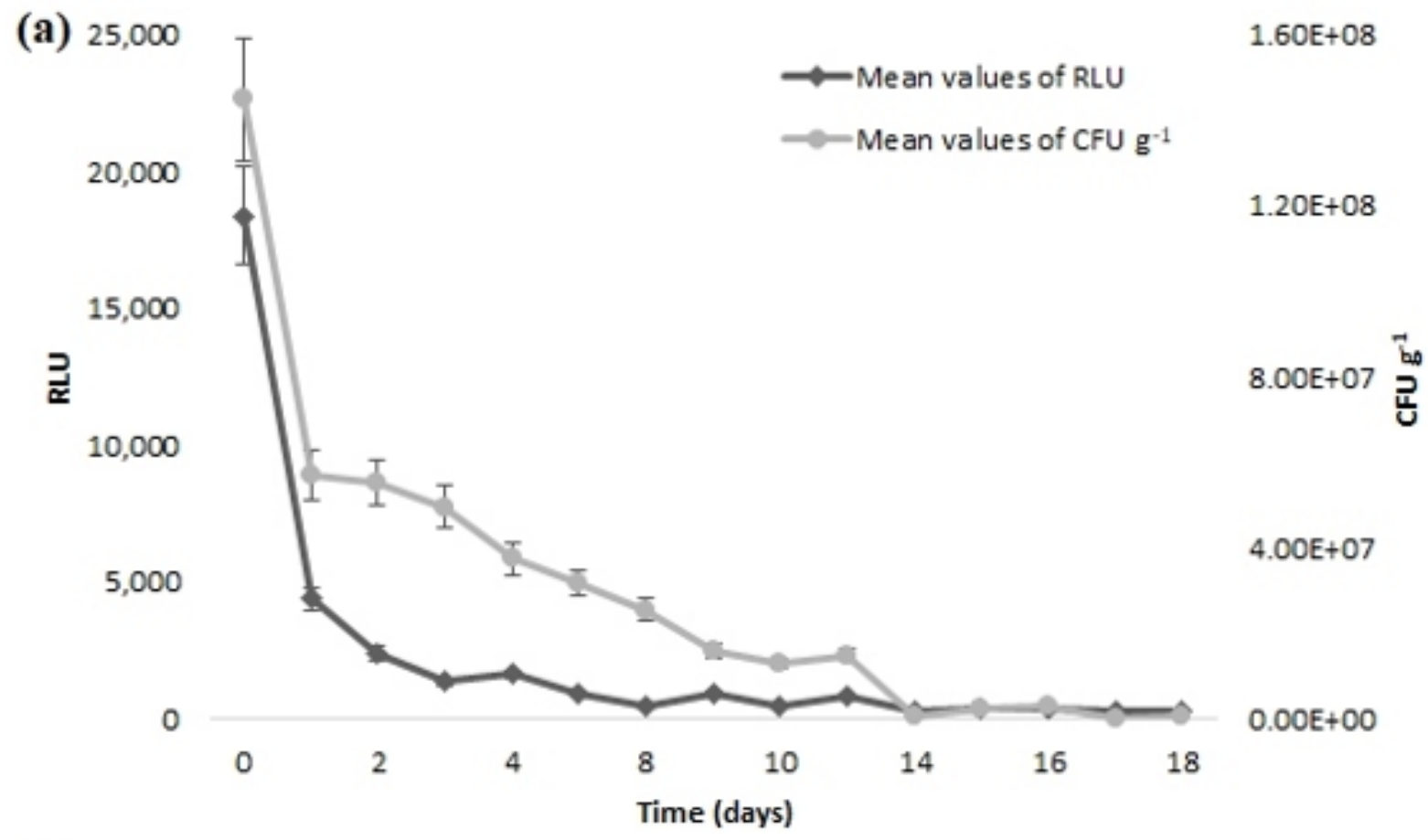

(b) 25,000

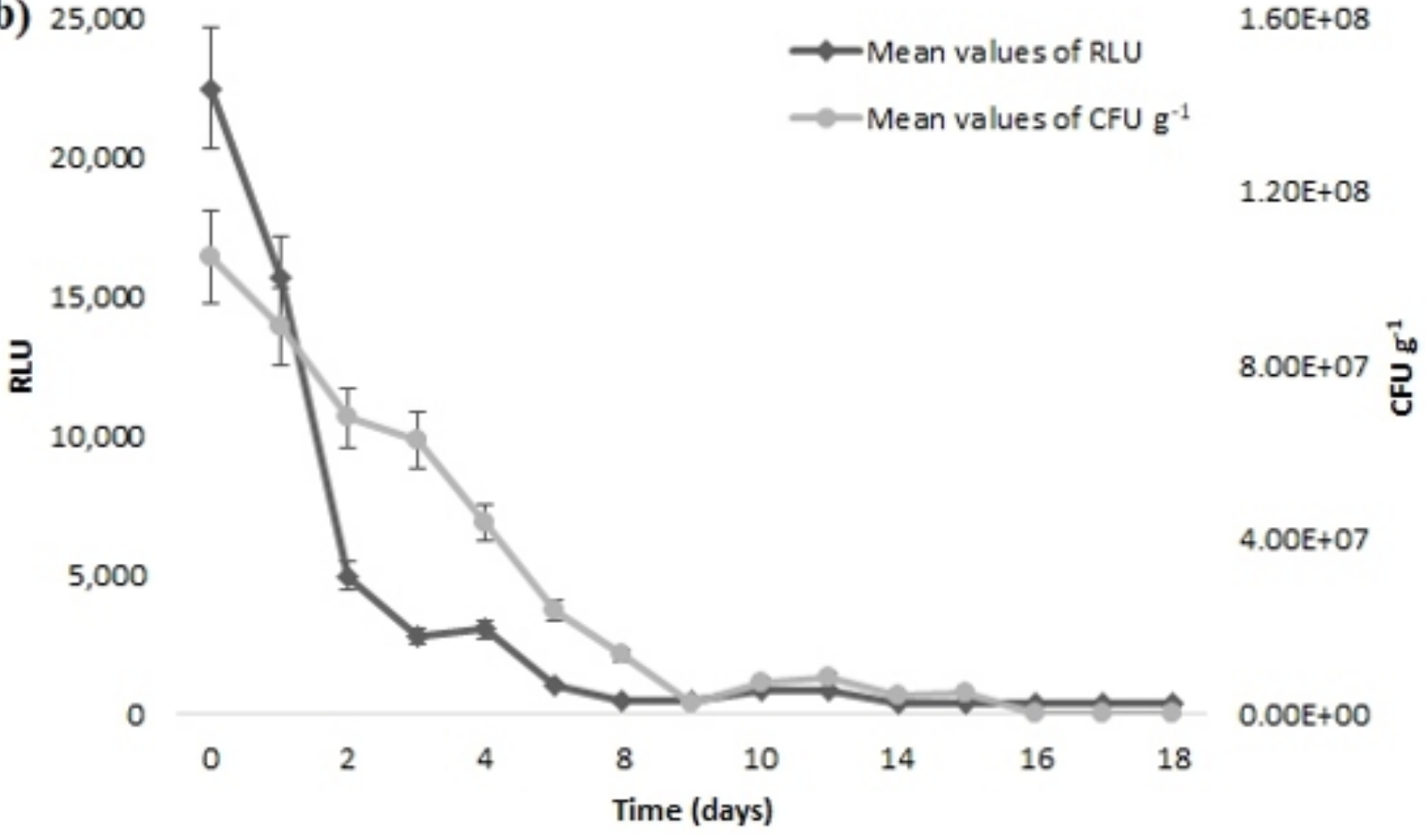

Figure 4: RLU and CFU g ${ }^{-1}$ counts of Tzatziki, (a) with and (b) without preservatives (Ringer solution) 
(a)

20,000

- Mean values of RLU

$1.20 \mathrm{E}+06$

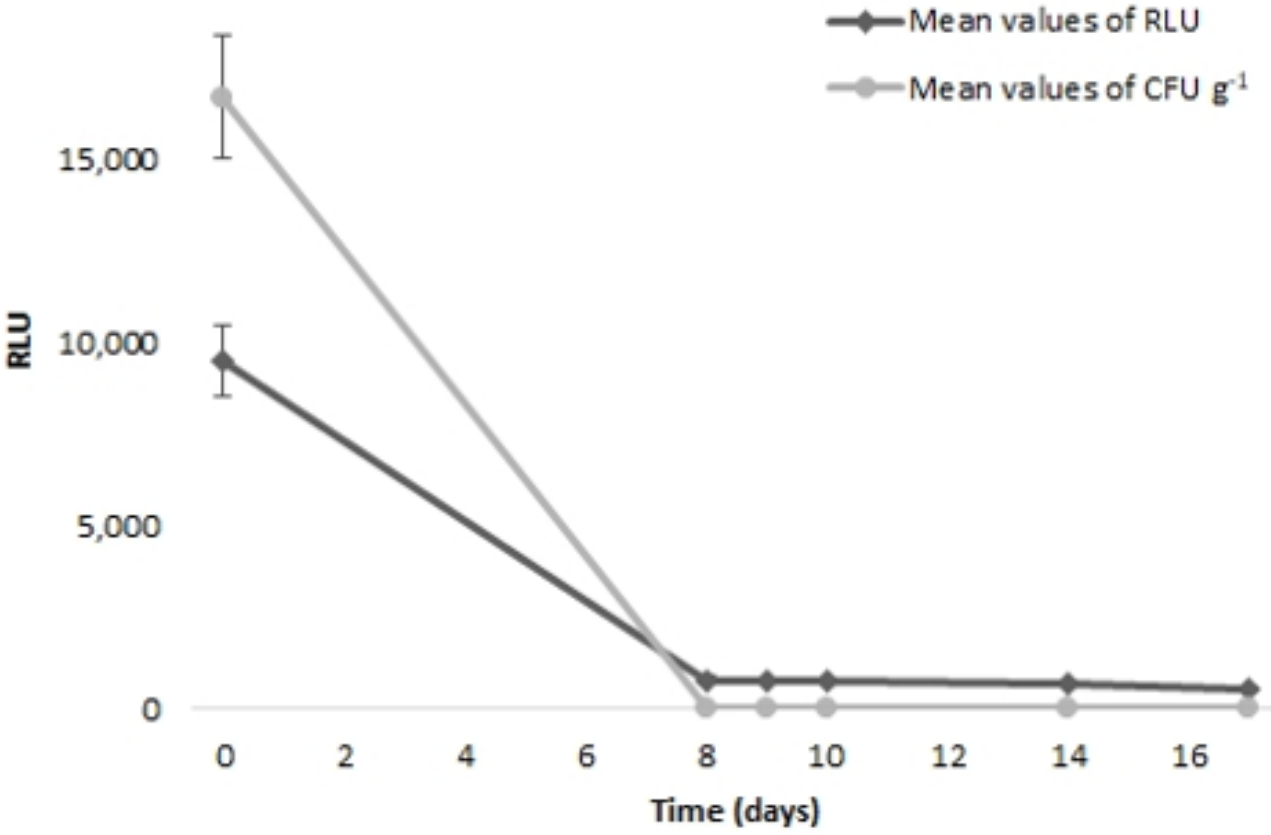

8.00E+05

$0.00 E+00$

(b) 20,000

$1.20 E+06$

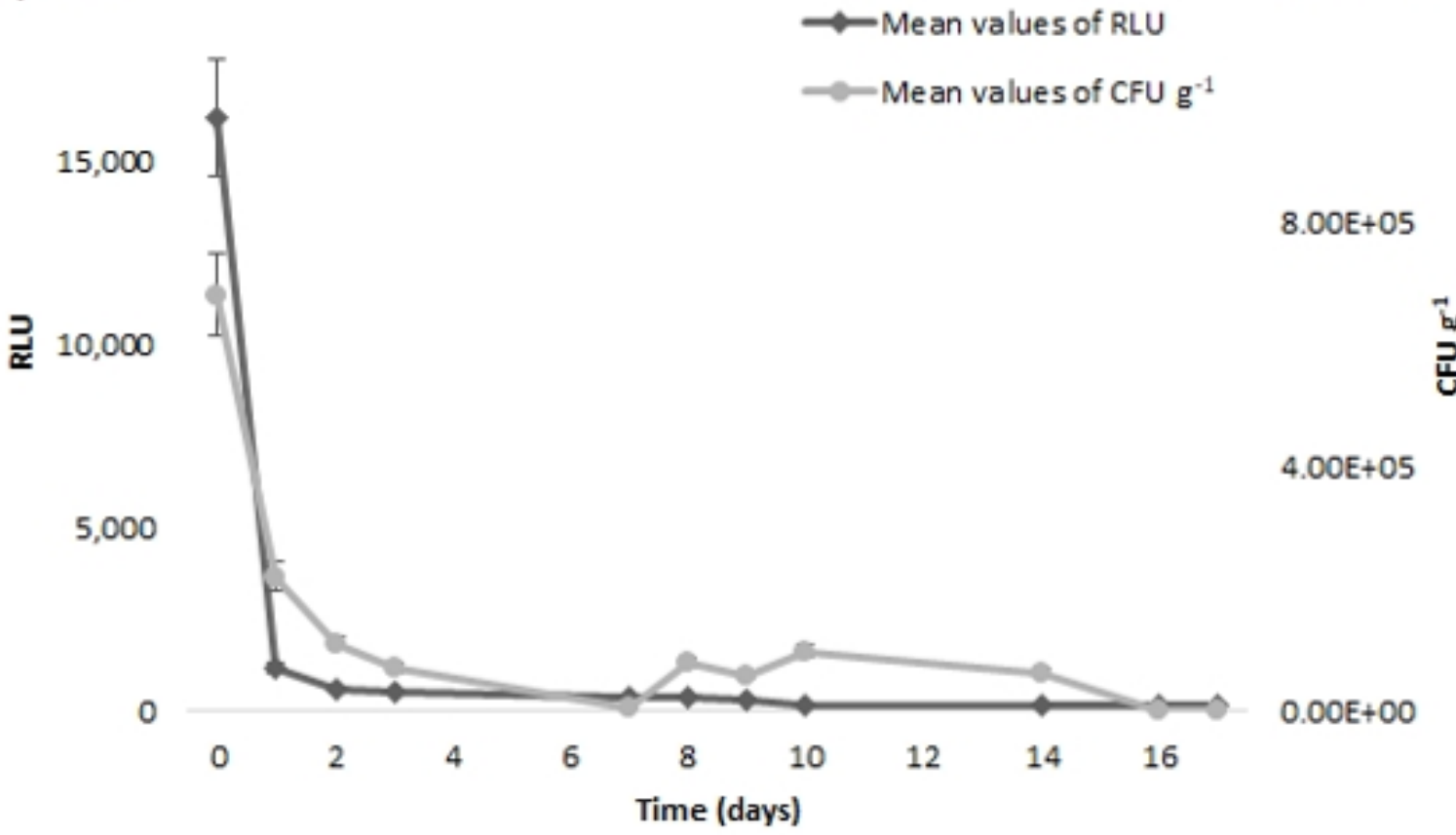

Figure 5: RLU and CFU $\mathrm{g}^{-1}$ counts of Tzatziki with preservatives incubated in (a) $\mu$ acConkey and in (b) Sabouraud for $24 \mathrm{~h}$ at $35^{\circ} \mathrm{C}$ 
(Kim, Huh, Kyung, \& Kyung, 2004). Cucumber is mainly responsible for the "fresh-green" aroma of fresh vegetables, which characterizes Tzatziki. Aroma compounds are plant secondary volatiles, which are produced when cucumber is finely chopped in order to be incorporated into the yogurt. Secondary volatiles, apart from their sensory contribution, can influence the microbial stability depending on their concentration, composition and type of plant material of origin. In some cases, the enzymatically formed secondary volatiles can accumulate to concentrations which are sufficient to decrease the microbial population. There are examples, such as the case of cucumber, where the main characteristic aroma predominates only when the enzymes are allowed to react on decompartmentation of the plant tissue, by cutting and homogenizing (Schrodter, 1984). Green aldehydes and green alcohols are the compounds responsible for the green flavor note. They are produced from linolenic and linoleic acid in wounded plant tissues (Galliard, 1978; Tressl \& Drawert, 1973). A large number of enzymes are released after cell membrane damage (Galliard, 1978). Autolysis gives free linolenic and linoleic acid. Lipoxygenase action on the above-mentioned acids gives rise to linolenic and linoleic hydroperoxides. The two hydroperoxides have antimicrobial activity (Brooks \& Watson, 1985). Lyase action on the hydroperoxides is responsible for the formation of (E)-2-Hexenal, $(E)$-2-Nonenal and(E,Z)-2,6Nonadienal. These compounds are the basis of green natural aroma at low thresholds (0.07 $\left.\mu \mathrm{g} \mathrm{L}^{-1}\right)$. It has been proved that the abovementioned aldehydes also possess fungistatic action in the gas phase (Gueldner, Wilson, \& Heidt, 1985; Huhtanen \& Guy, 1984).

Sulfur-containing volatiles are normally formed during crushing of the plant material. In Allium plants, essential oils possess antimicrobial activity. This anti-yeast activity (Wills, 1956) is probably due to the high allicin content. The effect of allicin on triose-phosphate-dehydrogenase in microbial cell metabolism has been demonstrated by Barone and Tansey (1977). Inhibition of several sulfhydryl metabolic enzymes was observed. The biogenesis of allicin was summarized by Dourtoglou (1986). The precursors and the products possess antifungal activity (Conner
\& Beuchat, 1984; Huhtanen \& Guy, 1984). Generally, the preservation of such products is based mainly on their high acidity and on chemical preservatives employed to inhibit the growth of spoilage and pathogenic microorganisms. However, consumers demand reduced levels of additives in foods and consider their use undesirable. Although the safety of these items is supposed to be ensured primarily by their low pH, several pathogens, namely E. coli O157:H7, L. monocytogenes, and Salmonella spp., have been reported to survive or even grow in these foods. Usually, the pathogens in such products come from the raw ingredients as well as due to contamination from the processing environment and packaging operation (Panagou, Nychas, \& Sofos, 2013).

That is why good manufacturing practice must be followed in order to minimize any possible cross-contamination. In the marketplace and consumers' homes, Tzatziki salad must be kept refrigerated and handled with stringent hygienic practices to prevent cross-contamination by other food products or the environment.

As indicated by the results, chemical preservatives are not essential for the microbial stabilization of the product. Specifically, after the $2^{\text {nd }}$ day, the Tzatziki salad showed its selfstabilization ability, even without the use of preservatives. Also, it was shown that an initial population of Lactobacillus spp. of $1.00 \mathrm{E}+08$ $\mathrm{CFU} \mathrm{g}^{-1}$ and RLU measurements lower than 4.00E $+04 \mathrm{RLU}$ in Greek traditional Tzatziki salad are considered as a minimum specification for the microbial stability and safety of the product.

Because of the good correlation that is presented between $\mathrm{CFU} \mathrm{g}^{-1}$ measurements and RLU values, the bioluminescence method appears to be an appropriate rapid method to determine Tzatziki microbial stability during industrial production practices. 


\section{Conclusions}

In conclusion, Greek traditional Tzatziki salad is a self-stable product due to its high acidity, plant derived natural preservatives and storage under refrigeration, and the rapid bioluminescence method can be used to define the microbial stability of the product from the beginning, on an industrial scale. The method requires little to no sample pretreatment as compared to conventional approaches and it can detect the total bacterial viability quickly and give an excellent aid in nonspecific detection of bacterial contaminants.

\section{Acknowledgements}

The authors would like to thank Mrs. Maria Alexandraki, technical personnel at Department of Food Technology for her guidance on the analysis by the bioluminescence method.

\section{References}

Barone, F. E., \& Tansey, M. R. (1977). Isolation, purification, identification, synthesis, and kinetics of activity of anti-candidal component of allium-sativum, and a hypothesis for its mode of action. Mycologia, 69(4), 793-825. doi:10.2307/3758870

Bottari, B., Santarelli, M., \& Neviani, E. (2015). Determination of microbial load for different beverages and foodstuff by assessment of intracellular atp. Trends in Food Science \& Technology, 44(1), 36-48. doi:10.1016/j. tifs.2015.02.012

Brooks, C. J. W., \& Watson, D. G. (1985). Phytoalexins. Natural Product Reports, 2(5), 427-459. doi:10.1039/np9850200427

Cho, M. J., Buescher, R. W., Johnson, M., \& Janes, M. (2004). Inactivation of pathogenic bacteria by cucumber volatiles (e,z)-2,6-nonadienal and (e)-2-nonenal. Journal of Food Protection, 67(5), 10141016. doi:10.4315/0362-028X-67.5.1014

Conner, D. E., \& Beuchat, L. R. (1984). Effects of essential oils from plants on growth of food spoilage yeasts. Journal of Food Sci- ence, 49(2), 429-434. doi:10.1111/j.13652621.1984.tb12437.x

Croft, K. P. C., Juttner, F., \& Slusarenko, A. J. (1993). Volatile products of the lipoxygenase pathway evolved from phaseolus-vulgaris (l) leaves inoculated with pseudomonas-syringae pvphaseolicola. Plant Physiology, 101(1), 13-24. doi:10.1104/pp.101.1.13

Dourtoglou, V. (1986). Production of natural flavourings, perfumes, and other fine chemicals by infected plants. Symbiosis (USA), 2, 189-200.

Galliard, T. (1978). Lipolytic and lipoxygenase enzymes in plants and their action in wounded tissues. Biochemistry of Wounded Plant Tissues, 155-201.

Gueldner, R. C., Wilson, D. M., \& Heidt, A. R. (1985). Volatile compounds inhibiting aspergillus-flavus. Journal of Agricultural and Food Chemistry, 33(3), 411-413. doi:10.1021/jf00063a022

Harris, J. C., Cottrell, S. L., Plummer, S., \& Lloyd, D. (2001). Antimicrobial properties of allium sativum (garlic). Applied Microbiology and Biotechnology, 57(3), 282-286. doi:10.1007/s002530100722

Hawronskyj, J. M., \& Holah, J. (1997). Atp: A universal hygiene monitor. Trends in Food Science \& Technology, 8(3), 79-84. doi:10. 1016/S0924-2244(97)01009-1

Huhtanen, C. N., \& Guy, E. J. (1984). Antifungal properties of esters of alkenoic and alkynoic acids. Journal of Food Science, $49(1), 281-$ \&. doi:10.1111/j.1365-2621.1984.tb13726.x

International Standard Organization. (1986). 5541-1 - Milk and milk products - Enumeration of coliforms - Part 1: Colony count technique at 30 degrees C. Geneva: BSI.

International Standard Organization. (2003a). 4833-1 - Microbiology of food and animal feeding stuffs - Horizontal method for the enumeration of microorganisms - Colonycount technique at 30 degrees C. Geneva: BSI.

International Standard Organization. (2003b). 9232 - Yogurt - Identification of characteristic microorganisms (Lactobacillus delbrueckii subsp. bulgaricus and Streptococcus thermophilus). Geneva: BSI. 
International Standard Organization. (2008). 21527-1 - Microbiology of food and animal feeding stuffs - Horizontal method for the enumeration of yeasts and moulds - Part 1: Colony count technique in products with water activity greater than 0,95. Geneva: BSI.

Kim, J. W., Huh, J. E., Kyung, S. H., \& Kyung, K. H. (2004). Antimicrobial activity of alk(en)yl sulfides found in essential oils of garlic and onion. Food Science and Biotechnology, 13(2), 235-239.

Luo, J., Liu, X., Tian, Q., Yue, W., Zeng, J., Chen, G., \& Cai, X. (2009). Disposable bioluminescence-based biosensor for detection of bacterial count in food. Analytical Biochemistry, 394 (1), 1-6. doi:10.1016/j. ab.2009.05.021

Mendonça, A. F., Juneja, V. K., \& Daraba, A. (2014). Total viable counts - metabolic activity tests. Elsevier.

Panagou, E. Z., Nychas, G.-J. E., \& Sofos, J. N. (2013). Types of traditional greek foods and their safety. Food Control, 29(1), 3241. doi:10.1016/j.foodcont.2012.05.050

Schrodter, R. (1984). Chromatographic studies of biogenesis of plant volatiles $(\mathrm{H}$. Heidelberg, Ed.).

Skandamis, P. N., Davies, K. W., McClure, P. J., Koutsoumanis, K., \& Tassou, C. (2002). A vitalistic approach for non-thermal inactivation of pathogens in traditional greek salads. Food Microbiology, 19(5), 405-421. doi:10.1006/yfmic.495

Sotiroudis, G., Melliou, E., Sotiroudis, T. G., \& Chinou, I. (2010). Chemical analysis, antioxidant and antimicrobial activity of three greek cucumber (cucumis sativus) cultivars. Journal of Food Biochemistry, 34 (s1), 61-78. doi:10.1111/j.1745-4514. 2009.00296.x

Tressl, R., \& Drawert, F. (1973). Biogenesis of banana volatiles. Journal of Agricultural and Food Chemistry, 21 (4), 560-565. doi:10.1021/jf60188a031

Tsiraki, M. I., \& Savvaidis, I. N. (2014). Citrus extract or natamycin treatments on "tzatziki"-a traditional greek salad. Food Chemistry, 142, 416-422. doi:10.1016/j . foodchem.2013.07.087
Wills, E. D. (1956). Enzyme inhibition by allicin, the active principle of garlic. Biochemical Journal, 63(3), 514-520. doi:10. 1042/bj0630514 